\title{
Suicídio em Mato Grosso - Brasil: 1996 a 2015
}

\section{Suicide in Mato Grosso - Brazil: 1996 to 2015}

\author{
Ligia Regina de Oliveira ${ }^{1,2}$ (D), Ariane de Oliveira Camargo Benedetti ${ }^{3,4}$ (D)
}

1. Docente do Departamento de Saúde Coletiva do Instituto de Saúde Coletiva da Universidade Federal de Mato Grosso (UFMT), Cuiabá, MT, Brasil. 2. Pósdoutora pela Faculdade de Saúde Pública da Universidade de São Paulo (USP), São Paulo, SP, Brasil. 3. Secretaria Municipal de Saúde de Lucas do Rio Verde, Lucas do Rio Verde, MT, Brasil. 4. Mestre em Psicologia e Sociedade pela Universidade Estadual Paulista "Júlio de Mesquita Filho" (UNESP), Assis, SP, Brasil.

\section{Resumo}

Introdução: 0 suicídio é uma das dez principais causas de mortalidade no mundo; é considerado um grande problema de saúde pública seja por sua magnitude, complexidade, seja pelo forte impacto social, econômico e pessoal. 0 Brasil é o oitavo país em número de suicídios, com média de 24 suicídios por dia. Objetivo: descrever a mortalidade por suicídios em Mato Grosso, no período de 1996 a 2015. Métodos: Realizou-se um estudo descritivo a partir de dados secundários do Sistema de Informações sobre Mortalidade/Ministério da Saúde. Os dados foram analisados em termos de frequência relativa (proporções, razão e taxas). Resultados: Os resultados revelam estabilidade da taxa de suicídio em Mato Grosso - média 6,8 óbitos/100.000 habitantes, mas com valores superiores ao Brasil - média 5,7 óbitos/100.000 habitantes. Observa-se a sobremortalidade masculina (Razão de sexo = 3,47), e taxas de suicídio mais elevadas em idosos $(9,8 / 100.000$ habitantes). As lesões autoprovocadas prevaleceram em relação à autointoxicação como método utilizado para o suicídio em uma razão de 4,83; porém, com comportamento diferente entre os sexos: 6,69 e 2,11, para o sexo masculino e feminino respectivamente. Entre as lesões autoprovocadas, enforcamento $(62,6 \%)$ e arma de fogo $(24,6 \%)$ foram as mais frequentes, enquanto as intoxicações por pesticidas (56,4\%) predominaram entre as autointoxicações. Conclusão: 0 estudo revela informações pouco exploradas sobre o suicídio no estado de Mato Grosso e, portanto, relevantes para ampliar o conhecimento sobre o problema e sua prevenção.

Palavras-chave: Suicídio. Epidemiologia. Mortalidade.

\begin{abstract}
Introduction: Suicide is one of the ten leading causes of mortality worldwide and considered a major problem for public health either due to its magnitude, complexity or by a strong social, economic and personal impact. Brazil is the eighth country in number of suicides, with an average of 24 suicides a day. Objective: describing mortality due to a suicide case in Mato Grosso from 1996 to 2015. Methods: A descriptive study from secondary data of the Mortality Data System/Ministry of Health was carried out. The data were analyzed in terms of relative frequency (proportions, ratio and rates). Results: The results show stability of the suicide rate in Mato Grosso - mean 6.8 deaths $/ 100,000$ inhabitants, but with values higher than in Brazil - 5.7 deaths/100,000 inhabitants. Excessive male mortality and higher suicide rates in the elderly $(9,8 / 100,000)$ have been noted. Self-inflicted injuries prevailed in relation to intentional intoxication as a method used for suicide in a ratio of 4.83 , however with different behavior between genders: 6.69 and 2.11 , for males and females respectively. Among the self-inflicted injuries, hanging (62.6\%) and firearm (24.6\%) were the most frequent, while pesticide poisonings (56.4\%) predominated among autointoxications. Conclusion: The study reveals little explored information on the suicide in the state of Mato Grosso and, hence, relevant to increase the understanding of the problem and its prevention.
\end{abstract}

Key words: Suicide. Epidemiology. Mortality.

\section{INTRODUÇÃO}

Com aproximadamente um milhão de mortes ao ano, o suicídio é uma das dez principais causas de mortalidade no mundo; ele é considerado um grande problema de saúde pública seja por sua magnitude, complexidade seja pelo forte impacto social, econômico e pessoal ${ }^{1-4}$.

O suicídio pode ser classificado como uma ação intencional iniciada e finalizada por uma pessoa que tem consciência e conhecimento do resultado de seu ato ${ }^{5}$. Contudo, sua definição é complexa e envolve interações e estudos de várias áreas do conhecimento: filosofia, antropologia, psicologia, biologia e ciências sociais ${ }^{5}$.

São diversos os fatores envolvidos na gênese do suicídio, entre eles, os problemas biológicos, médicos, ambientais, psiquiátricos e psicológicos, filosóficos-existenciais e motivações sociais ${ }^{1,4,6-8}$.

A desigualdade social, a baixa renda, o desemprego e a escolaridade também são fatores relacionados à ocorrência do suicídio, o que contribui para que a distribuição do suicídio no mundo não seja homogênea ${ }^{4,7,8}$. Segundo a Organização Mundial da Saúde (OMS) 4, 75\% dos casos de suicídio se dão em países subdesenvolvidos ou em desenvolvimento, apontando para uma correlação entre situação econômica e taxas de suicídio. Em 2012, a taxa de mortalidade por suicídio no mundo foi de $11,4 / 100.000$ habitantes. No entanto, sua distribuição se mostrou heterogênea, sendo a taxa mais elevada encontrada na Ásia $(17,7 / 100.000$ habitantes), vindo, a seguir, a Europa (12,0/100.000 habitantes) enquanto as Américas apresentavam

Correspondência: Ligia Regina de Oliveira. Universidade Federal de Mato Grosso Av. Fernando Corrêa da Costa, 2367 - Boa Esperança, Cuiabá - MT, 78060 900. E-mail: Irdoliveira@gmail.com

Conflito de interesse: Não há conflito de interesse por parte de qualquer um dos autores.

Recebido em: 17 Set 2017; Revisado em: 6 Jun 2018; 9 Ago 2018; Aceito em: 10 Ago 2018 
taxa de mortalidade de 6,1/100.000 habitantes ${ }^{4}$.

Historicamente, o suicídio é mais frequente em homens que mulheres ${ }^{1,2,4,8,9}$. A taxa global de suicídio entre os homens é de 15,0 por 100.000 habitantes e, entre as mulheres, é de $8,0^{4}$. Em relação à idade, o suicídio é a segunda causa de morte no grupo etário de 15 a 29 anos, sendo este o grupo de maior risco 4 . Entre 1950 e 2000, os jovens de 15 a 44 anos passaram a se suicidar mais que os adultos com idade acima de 45 anos, e essa tendência parece manter-se nos próximos anos, segundo projeções da $\mathrm{OMS}^{2}$.

A prevalência de pessoas portadoras de algum transtorno mental, seja depressão, esquizofrenia, transtorno do humor, entre outros, tem sido apontada como um importante fator na epidemiologia do suicídio ${ }^{8,10-12}$. Um estudo de revisão ${ }^{10}$ verificou que, em mais de $90 \%$ dos casos de suicídio, caberia um diagnóstico de transtorno mental. Entre outros fatores considerados dispositivos para o suicídio, estão alguns eventos da vida que afetam, emocionalmente, o indivíduo, como perdas de entes queridos, problemas no trabalho, conflitos conjugais, dificuldade de relacionamento interpessoal e solidão ${ }^{1}$. Para $\mathrm{OECD}^{8}$, o suicídio pode ser utilizado como um indicador indireto do estado de saúde mental da população.

$\mathrm{A} \mathrm{OMS}^{4}$ estima que a quantidade de tentativas é de 10 a 20 vezes mais alta que a de mortes. Um fator de destaque, na epidemiologia do suicídio, é a tendência 20 vezes maior de tentativas de suicídio do sexo feminino que a do masculino; porém, há diferença de métodos escolhidos, e, nos homens, a eficácia se mostra maior ${ }^{5,13,14}$. O enforcamento e o uso de armas de fogo são os principais métodos para o suicídio, principalmente nos países de alta renda das Américas enquanto o uso de pesticidas se destaca em países de baixa ou média renda ${ }^{4}$. Para Botega ${ }^{11}$, os meios utilizados variam conforme a cultura e a disponibilidade.

O Brasil é o oitavo país em número de suicídios, com média de 24 suicídios por dia ${ }^{1}$. Entre 2000 e 2012, houve aumento de $26,5 \%$ na mortalidade por suicídio no país, passando de 4,9/100.000 habitantes para 6,2, sendo esta causa de morte 3,7 vezes mais frequente em homens do que em mulheres ${ }^{7}$.

Importante destacar que um coeficiente nacional de mortalidade por suicídio pode esconder importantes variações regionais ${ }^{6,11,15}$. Em 2013, a Região Sudeste apresentou a maior proporção de mortes por suicídio (37,6\%); no entanto, foi a Região Sul a que obteve a maior taxa de mortalidade $(8,2 / 100.000 \text { habitantes })^{1}$. Por outro lado, ressalta-se que o crescimento de mortes por suicídio, no período de 2000 a 2012, foi maior nas regiões Norte e Nordeste do país ${ }^{15}$.

Em 2013, do total de suicídios ocorridos no Brasil, 42,6\% ocorreram na população de 20 a 39 anos $^{1}$. Estudos recentes têm revelado que, no país, as taxas mais elevadas são encontradas em homens, idosos, indígenas e em cidades de pequeno e de médio porte populacional ${ }^{16,17}$.

Contudo, assim como em todo o mundo, a mortalidade por suicídio no Brasil pode ser ainda maior tendo em vista a subnotificação, decorrente do estigma social que favorece a omissão de $\operatorname{casos}^{4,11}$. Para além do sub-registro ou da subnotificação, alguns autores afirmam que os suicídios também se "escondem" sob outras denominações de causas de morte acidentais (acidente automobilístico, afogamento, envenenamento acidental) e também sob as "causas indeterminadas" ${ }^{18,19}$.

Sendo o suicídio um grave problema de saúde pública e considerando a complexidade que envolve o tema, a prevenção do comportamento suicida não é uma tarefa fácil20,21. No entanto, a OMS afirma que a prevenção do suicídio é factível, mas requer diferentes estratégias que abrangem a atenção à saúde e o controle dos fatores de risco ${ }^{3,4}$. A observação à questão socioeconômica e educacional da população também se revela como importante estratégia na prevenção desse agravo ${ }^{4,14}$. Igualmente, o aumento da percepção e a disseminação de informação apropriada são elementos essenciais para os programas de prevenção do suicídio ${ }^{21}$.

Tendo em vista o grande número de casos de suicídio ligados a pessoas com transtornos mentais, a prevenção, em princípio, tem relação com a identificação precoce e o correto encaminhamento de casos de transtornos mentais ${ }^{22,23}$. Atenção especial à pessoa que tentou se suicidar é uma das principais estratégias de prevenção do suicídio ${ }^{4,11,21,23,24}$. Nesse sentido, ressalta-se a qualificação dos profissionais como primordial para a detecção precoce e a prevenção ${ }^{20-23}$. Contudo, a falta de capacitação ${ }^{25-27}$ assim como a formação dos profissionais da área biomédica ${ }^{26}$ têm sido indicativos importantes para a dificuldade de lidar com o ato suicida.

Ainda no sentido de prevenção, a $\mathrm{OMS}^{4}$ indica algumas estratégias para dificultar o ato, como o limite ao acesso às armas de fogo, aos pesticidas e a certos medicamentos que estão entre os métodos mais utilizados para o suicídio.

A prevenção do suicídio deve ser um dos eixos centrais dos serviços de assistência à saúde ${ }^{4}$, portanto uma rede de saúde mental efetiva que atenda aos usuários de saúde mental em todos os níveis de assistência é essencial ${ }^{11,22,23}$. No Brasil, as Diretrizes Nacionais de Prevenção ao Suicídio, instituídas em $2006^{28}$, destacam a importância da estruturação da rede de saúde mental e apontam outras ações de prevenção, como o estímulo a pesquisas sobre o tema, o aprimoramento da notificação dos casos de suicídios e tentativas, a redução do acesso a meios letais e a promoção da educação permanente dos profissionais de saúde.

Contudo, para que as ações de prevenção e de atenção conquistem êxito, é necessário se conhecer e debater mais sobre o tema no Brasil. Além dos dados globais do país, considerando 
a importância do suicídio, sua complexidade, as perspectivas de ascendência deste e a possibilidade de prevenção, é preciso desvendar características e fatores de risco específicos para as diferentes regiões do país ${ }^{1,11,15}$ permitindo, dessa forma, o melhor delineamento de estratégias de enfrentamento.

Posto isso, e considerando a escassez de pesquisas epidemiológicas sobre suicídio no estado de Mato Grosso, é que este estudo objetiva descrever a mortalidade por suicídios no estado no período de 1996 a 2015.

\section{MÉTODOS}

Os dados se referem às mortes por suicídio, ocorridas no período de 1996 a 2015, em indivíduos com 10 anos ou mais de idade, residentes no estado de Mato Grosso-Brasil. Foram considerados todos os óbitos classificados sob os códigos $X 60$ a X84 da Classificação Estatística Internacional de Doenças e Problemas Relacionados à Saúde: 10a revisão (CID-10) ${ }^{29}$.

Realizou-se um estudo descritivo a partir de dados secundários do Sistema de Informações sobre Mortalidade obtidos no site do DATASUS/Ministério da Saúde ${ }^{30}$ e da Secretaria de Estado da Saúde ${ }^{31}$ para o ano de 2015 , tendo em vista que não há dados para esse ano no site do DATASUS. Dados populacionais, utilizados para o cálculo das taxas de mortalidade, foram também coletados no site do DATASUS.

Os dados foram analisados em termos de frequência relativa (proporções, razão e taxas). A análise proporcional foi estratificada segundo o sexo, e, para os cálculos percentuais, foram excluídos os dados "ignorados".

Analisou-se a incidência de suicídio por sexo e faixa etária (10 a 19 anos, 20 a 29 anos, 30 a 39 anos, 40 a 49 anos, 50 a 59 anos, 60 anos e mais) e a mortalidade proporcional por raça/cor (branca, parda/preta, amarela, indígena), escolaridade (nenhuma, 1 a 7 anos, 8 a 11 anos, 12 anos e mais de estudo), estado civil (divorciado/solteiro, casado, viúvo, outros) e local de ocorrência (hospital/serviço de saúde, via pública, domicílio). Foi ainda analisada a mortalidade proporcional segundo método ou meio utilizado para concretização do suicídio, destacando-se os dois grupamentos: i) Autointoxicações intencionais (X60-X69) e ii) Lesões autoprovocadas intencionalmente (X70-X84). Esses ainda foram agrupados nas categorias: medicamentos diversos (X60, X61, X63 e X64); narcóticos, psicodislépticos NCOP (X62); álcool (X65); outros gases e vapores (X67); pesticidas (X86); outros produtos químicos, substâncias nocivas não especificadas (X69); enforcamento, estrangulamento, sufocamento (X70); afogamento, submersão (X71); arma de fogo (X72-X74); fumaça, fogo e chamas (X76, X77); objeto cortante, penetrante, contundente $(X 78, X 79)$; precipitação de lugar elevado (X80); outros meios especificados (X81-X83) e meios não especificados (X84). Para esses, foi estimado o intervalo de confiança de 95\% (IC95\%).

Considerando que os dados analisados são de acesso público e, portanto, estão omissas informações que possam identificar o sujeito, este estudo atende aos princípios de ética em pesquisa envolvendo seres humanos, em conformidade com a Resolução no 466, de 12 de dezembro de 2012, do Conselho Nacional de Saúde.

\section{RESULTADOS}

No período entre 1996 e 2015 foram registrados 3.051 suicídios na população com 10 anos ou mais residente no estado de Mato Grosso. Prevaleceu o sexo masculino $(78,4 \%)$, e a razão entre o número de suicídio de homens e mulheres foi de 3,6, variando de 2,3 (2002) a 5,7 (2003).

A taxa de mortalidade por suicídio em Mato Grosso pouco oscilou nos vinte anos de estudo, apresentando média de 6,8 óbitos/100.000 habitantes para a população de 10 anos ou mais, valor mais elevado que o encontrado para o Brasil (19962014) - 5,7 óbitos/100.000 habitantes. Em homens, a taxa encontrada para o período foi de 10,4, e, em mulheres, de 3,0, com razão de sexo 3,47. Em todos os anos, a taxa de suicídios se manteve sempre mais elevada no sexo masculino, apresentando a maior taxa em 2003 e 2009 com 12,7 óbitos/100.000; para as mulheres, a taxa mais elevada se deu em 2002 com 4,6 óbitos/100.000 (Figura 1).

Figura 1. Taxa de suicídios (Óbitos/100.000 habitantes) segundo o sexo. Mato Grosso, 1996 a 2015.

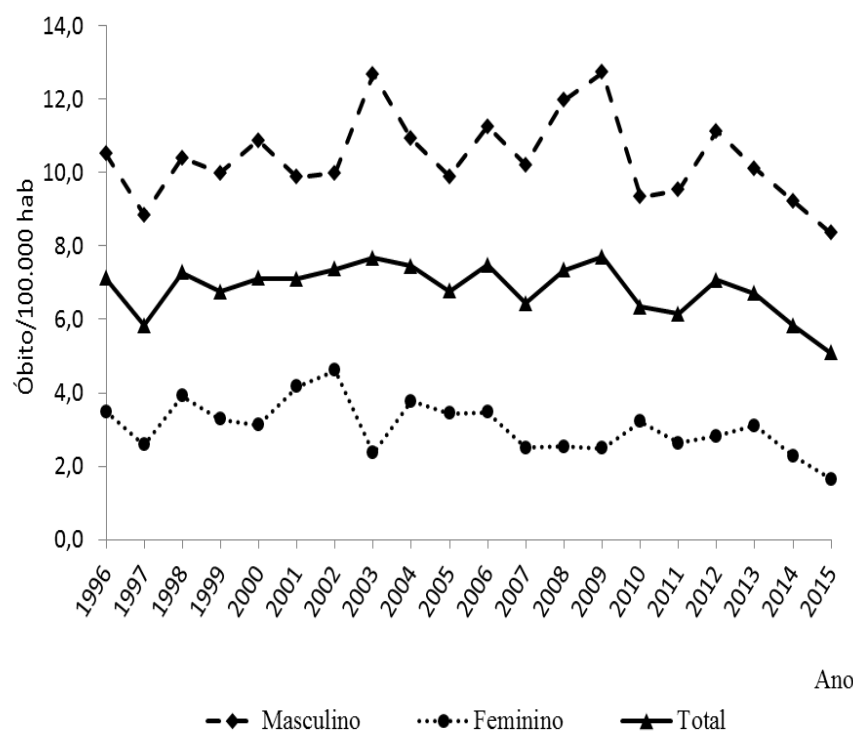

Os dados revelam que, no período estudado, as lesões autoprovocadas prevaleceram em relação à autointoxicação como meio ou método utilizado para o suicídio, em uma relação de 4,83, porém com comportamento diferente entre os sexos: 6,69 e 2,11 para o sexo masculino e feminino respectivamente. Predominaram entre as lesões autoprovocadas, o enforcamento $(62,6 \%$; IC95\% 60,7-64,5) e a arma de fogo (24,6\%; IC95\% 22,9$26,3)$, e para a autointoxicação, o uso de pesticidas $(56,4 \%$; IC95\% 52,0-60,7) e de medicamentos diversos (25,0\%; IC95\% 21,4-29,0), como observado na tabela 1 . 
Tabela 1. Suicídios segundo o meio utilizado e o sexo. Mato Grosso, 1996 a 2015.

\begin{tabular}{|c|c|c|c|c|c|c|}
\hline \multirow{3}{*}{$\begin{array}{l}\text { Meio utilizado / } \\
\text { Categoria (CID10) }\end{array}$} & \multicolumn{4}{|c|}{ SEXO } & \multirow{2}{*}{\multicolumn{2}{|c|}{ Total }} \\
\hline & \multicolumn{3}{|c|}{ Masculino } & \multirow{2}{*}{$\begin{array}{r}\text { Feminino } \\
\text { IC } 95 \%\end{array}$} & & \\
\hline & $\%$ & IC 95\% & $\%$ & & $\%$ & IC $95 \%$ \\
\hline Autointoxicação & 311 & & 212 & & 523 & \\
\hline Medicamentos diversos & 19,0 & $14,8-23,8$ & 34,0 & $27,6-40,8$ & 25,0 & $21,4-29,0$ \\
\hline Narcóticos, psicodislept NCOP ${ }^{1}$ & 1,3 & $0,4-3,3$ & 0,5 & $0,0-2,6$ & 1,0 & $0,3-2,2$ \\
\hline Álcool & 1,0 & $0,2-2,8$ & 1,4 & $0,3-4,1$ & 1,1 & $0,4-2,5$ \\
\hline Outros gases e vapores & 1,3 & $0,4-3,3$ & 0,5 & $0,0-2,6$ & 1,0 & $0,3-2,2$ \\
\hline Pesticidas & 60,8 & $55,1-66,2$ & 50,0 & $43,1-56,9$ & 56,4 & $52,0-60,7$ \\
\hline Outros prod. químicos, $\mathrm{NE}^{2}$ & 16,7 & $12,7-21,3$ & 13,7 & $9,4-19,1$ & 15,5 & $12,5-18,9$ \\
\hline Lesões autoprovocadas & 2.081 & & 447 & & 2.528 & \\
\hline Enforcamento, estrangulamento, sufocamento & 61,8 & $59,7-64,0$ & 66,0 & $61,4-70,4$ & 62,6 & $60,7-64,5$ \\
\hline Afogamento submersão & 1,4 & $1,0-2,1$ & 1,1 & $0,4-2,6$ & 1,4 & $1,0-1,9$ \\
\hline Arma de fogo & 26,4 & $24,5-28,3$ & 16,3 & $13,2-20,1$ & 24,6 & $22,9-26,3$ \\
\hline Fumaça, fogo e chamas & 0,6 & $0,3-1,0$ & 6,7 & $4,6-9,4$ & 1,7 & $1,2-2,2$ \\
\hline Objeto corta., penetr., contundente & 6,0 & $5,0-7,1$ & 3,6 & $2,1-5,7$ & 5,6 & $4,7-6,5$ \\
\hline Precipitação de lugar elevado & 1,3 & $0,9-1,9$ & 3,1 & $1,7-5,1$ & 1,6 & $1,2-2,2$ \\
\hline Outros meios especificados & 0,9 & $0,5-1,4$ & 0,9 & $0,2-2,3$ & 0,9 & $0,5-1,3$ \\
\hline Meios não especificados & 1,6 & $1,1-2,2$ & 2,2 & $1,1-4,1$ & 1,7 & $1,2-2,3$ \\
\hline Total & 2.392 & & 659 & & 3.051 & \\
\hline
\end{tabular}

${ }^{1}$ Não classificado em outra parte.

${ }^{2}$ Não especificados

No que se refere aos suicídios causados por autointoxicação, no sexo masculino, os pesticidas representaram 60,8\% (IC95\% 55,1$66,2)$ enquanto, entre as mulheres, esses foram responsáveis por metade das mortes; já os medicamentos estiveram relacionados com $19,0 \%$ (IC95\% 14,8-23,8) das mortes no sexo masculino e $34,0 \%$ (IC95\% 27,6-40,8) no sexo feminino (Tabela 1).

Para o sexo masculino, o enforcamento representou $61,8 \%$ (IC95\% 59,5-64,0) das lesões autoprovocadas e a arma de fogo, 26,4\% (IC95\% 24,5-28,2); entre as mulheres, esses percentuais foram de $66,0 \%$ (IC95\% 61,4-70,4) e 16,3\% (IC95\% 13,2-20,1) respectivamente (Tabela 1 ). Ainda em relação às lesões autoprovocadas, chama atenção o percentual de mortes ocorridas em mulheres cujo método de escolha foi "fumaça/ fogo/chama" (6,7\%; IC95\% 4,6-9,4) quando comparado às proporções encontradas para o sexo masculino $(0,6 \%$; IC95\% $0,3-1,0)$.

Cerca de metade $(48,1 \%)$ das vítimas era adulto com idade entre 20 a 39 anos (Tabela 2). Em homens, a proporção de suicídios em idosos $(13,2 \%)$ é mais elevada quando comparada com a proporção em mulheres $(7,4 \%)$. Diversamente, no sexo feminino, a proporção de suicídios em adolescentes é bastante superior $(18,2 \%)$ à do sexo masculino $(8,3 \%)$.

A raça preta/parda foi prevalente $(60,5 \%)$ em ambos os sexos, e em relação ao estado civil; indivíduos separados ou solteiros foi a maioria (56,6\%). Quanto à escolaridade, 70,0\% tinham baixa escolaridade (nenhuma ou menos que 7 anos de estudo). A comparação entre os sexos mostra diferença na distribuição por escolaridade. Para o sexo feminino, o percentual de indivíduos com escolaridade de 12 anos ou mais de estudo foi $11,8 \%$ e, para o sexo masculino, esse grupo representou $8,7 \%$; enquanto nenhuma escolaridade foi mais prevalente no sexo masculino $(17,0 \%)$ que no feminino $(11,1 \%)$ (Tabela 2$)$.

Menos de $1 / 5$ dos suicídios (19,3\%) se deu no ambiente hospitalar ou em outros estabelecimentos de saúde (Tabela 2 ), prevalecendo o domicílio como local de ocorrência $(58,1 \%)$. No entanto, destaca-se que $31,7 \%$ dos óbitos por suicídio no sexo feminino ocorreram em ambiente hospitalar ou em outros estabelecimentos de saúde.

Apesar do baixo percentual de idosos no total dos suicídios $(12,0 \%)$, a maior taxa de mortalidade no período se deu nesse grupo etário (9,8/100.000 habitantes), como observado na tabela 3. Em dez anos, dos vinte estudados, esse grupo etário apresentou a maior taxa de mortalidade, e em seis, a segunda maior taxa quando comparada com os demais grupos. O segundo grupo etário que apresentou as taxas mais elevadas foi o de 50 a 59 anos (8,7/100.000 habitantes). Em todos os anos, a taxa de mortalidade por suicídio foi menor em adolescentes, variando de 1,4/100.000 habitantes (2010) a 4,2/100.000 habitantes (2009). 
Tabela 2. Suicídios (número e percentual) segundo características das vítimas e sexo. Mato Grosso, 1996 a 2013.

\begin{tabular}{|c|c|c|c|c|c|c|}
\hline \multirow{3}{*}{ Características } & \multicolumn{4}{|c|}{ SEXO } & \multirow{2}{*}{\multicolumn{2}{|c|}{ Total }} \\
\hline & \multicolumn{2}{|c|}{ Masculino } & \multicolumn{2}{|c|}{ Feminino } & & \\
\hline & $n^{\circ}$ & $\%$ & $n^{\circ}$ & $\%$ & $n^{\circ}$ & $\%$ \\
\hline \multicolumn{7}{|l|}{ Faixa etária $(n=3.051)$} \\
\hline $10-19$ & 198 & 8,3 & 120 & 18,2 & 318 & 10,4 \\
\hline $20-29$ & 619 & 25,9 & 162 & 24,6 & 781 & 25,6 \\
\hline $30-39$ & 540 & 22,6 & 145 & 22,0 & 685 & 22,5 \\
\hline $40-49$ & 424 & 17,7 & 119 & 18,1 & 543 & 17,8 \\
\hline $50-59$ & 295 & 12,3 & 64 & 9,7 & 359 & 11,8 \\
\hline 60 e mais & 316 & 13,2 & 49 & 7,4 & 365 & 12,0 \\
\hline \multicolumn{7}{|l|}{ Raça/cor (n=2.719) } \\
\hline Preta/Parda & 1315 & 61,6 & 330 & 56,5 & 1645 & 60,5 \\
\hline Branca & 806 & 37,8 & 249 & 42,6 & 1055 & 38,8 \\
\hline Amarela & 4 & 0,2 & 3 & 0,5 & 7 & 0,3 \\
\hline Indígena & 10 & 0,5 & 2 & 0,3 & 12 & 0,4 \\
\hline \multicolumn{7}{|l|}{ Escolaridade $(n=2.193)$} \\
\hline Nenhuma & 204 & 17,0 & 53 & 11,1 & 257 & 11,7 \\
\hline 1 a 7 anos & 519 & 43,3 & 241 & 50,6 & 1279 & 58,3 \\
\hline 8 a 11 anos & 371 & 31,0 & 126 & 26,5 & 497 & 22,7 \\
\hline 12 anos e mais & 104 & 8,7 & 56 & 11,8 & 160 & 7,3 \\
\hline \multicolumn{7}{|l|}{ Estado Civil ( $n=2.751)$} \\
\hline Solteiro+Separado & 1254 & 58,1 & 303 & 51,3 & 1557 & 56,6 \\
\hline Casado & 698 & 32,3 & 197 & 33,3 & 895 & 32,5 \\
\hline Viúvo & 67 & 3,1 & 30 & 5,1 & 97 & 3,5 \\
\hline Outro & 141 & 6,5 & 61 & 10,3 & 202 & 7,3 \\
\hline \multicolumn{7}{|l|}{$\begin{array}{l}\text { Local de Ocorrência } \\
\text { (n=3.018) }\end{array}$} \\
\hline $\begin{array}{l}\text { Hospital }+{ }^{+} \text {outros } \\
\text { estabel. de saúde }\end{array}$ & 376 & 15,9 & 207 & 31,7 & 583 & 19,3 \\
\hline Domicílio & 1380 & 58,4 & 374 & 57,3 & 1754 & 58,1 \\
\hline Via pública & 143 & 6,0 & 24 & 3,7 & 167 & 5,5 \\
\hline Outros & 466 & 19,7 & 48 & 7,4 & 514 & 17,0 \\
\hline
\end{tabular}

\section{DISCUSSÃO}

Este estudo descreveu a mortalidade por suicídios no estado de Mato Grosso-Brasil entre o ano de 1996 e 2015, revelando que, apesar de estáveis, as taxas de mortalidade no período se comportaram diferentemente quando desagregadas segundo algumas variáveis. Destaca-se que, durante os anos de estudo, exceto 2014, a taxa de suicídio no estado apresentou valores superiores ao Brasil $^{1}$.

Os homens e os idosos foram os grupos de maior risco de suicídio em Mato Grosso. Maior prevalência foi observada em indivíduos de cor parda/preta, com pouca escolaridade e solteiros. Assim como no Brasil ${ }^{11}$, o domicílio destaca-se como o lugar de maior ocorrência do suicídio no estado.
Tabela 3. Taxa de suicídios (Óbitos/100.000 habitantes) segundo faixa etária. Mato Grosso, 1996 a 2013.

\begin{tabular}{|c|c|c|c|c|c|c|}
\hline \multirow{2}{*}{ Ano } & \multicolumn{6}{|c|}{ Faixa Etária (anos) } \\
\hline & 10 a 19 & 20 a 29 & 30 a 39 & 40 a 49 & 50 a 59 & 60 e mais \\
\hline 1996 & 3,7 & 7,8 & 8,2 & 6,9 & 12,6 & 10,7 \\
\hline 1997 & 2,7 & 6,9 & 6,9 & 5,0 & 13,1 & 6,1 \\
\hline 1998 & 3,0 & 6,4 & 8,4 & 10,6 & 13,6 & 12,9 \\
\hline 1999 & 3,1 & 6,0 & 11,9 & 7,4 & 5,2 & 11,1 \\
\hline 2000 & 2,7 & 9,7 & 6,9 & 11,9 & 7,0 & 6,9 \\
\hline 2001 & 3,4 & 8,7 & 10,4 & 5,8 & 5,6 & 10,9 \\
\hline 2002 & 2,1 & 7,9 & 8,3 & 12,2 & 10,5 & 10,8 \\
\hline 2003 & 2,8 & 10,7 & 7,0 & 9,5 & 8,5 & 13,9 \\
\hline 2004 & 2,9 & 9,0 & 7,1 & 9,3 & 11,3 & 13,1 \\
\hline 2005 & 3,8 & 5,6 & 8,6 & 8,0 & 10,4 & 10,8 \\
\hline 2006 & 2,7 & 6,1 & 7,8 & 14,1 & 10,2 & 14,4 \\
\hline 2007 & 2,5 & 6,6 & 6,5 & 9,7 & 11,3 & 5,4 \\
\hline 2008 & 3,3 & 8,5 & 7,4 & 8,1 & 9,0 & 11,6 \\
\hline 2009 & 4,2 & 8,8 & 9,1 & 6,6 & 10,6 & 9,3 \\
\hline 2010 & 1,4 & 6,7 & 6,2 & 9,5 & 8,0 & 10,4 \\
\hline 2011 & 2,1 & 6,7 & 6,3 & 6,9 & 8,3 & 10,3 \\
\hline 2012 & 2,8 & 7,8 & 9,9 & 7,3 & 6,3 & 9,8 \\
\hline 2013 & 2,7 & 7,1 & 6,1 & 10,2 & 7,4 & 9,2 \\
\hline 2014 & 2,3 & 7,4 & 7,7 & 5,3 & 6,1 & 6,5 \\
\hline 2015 & 2,5 & 5,4 & 5,3 & 4,8 & 6,8 & 7,6 \\
\hline Total & 2,8 & 7,5 & 7,7 & 8,3 & 8,7 & 9,8 \\
\hline
\end{tabular}

Historicamente, o suicídio é mais frequente em homens que mulheres $^{4,6,8}$. A Organização Mundial de Saúde informa que a razão entre a taxa de suicídio de homens e mulheres nos países desenvolvidos é de 3,5 e, nos países em desenvolvimento, é de $1,6^{4}$. Neste estudo, a razão encontrada foi semelhante à de países desenvolvidos e próxima aos resultados do Brasil ${ }^{1}$. Valor divergente foi identificado em indígenas, no qual a razão de sexo foi, em média, 5,0.

Vários pesquisadores afirmam que a eficácia dos métodos é maior nos homens que nas mulheres, daí esse grupo obter maior sucesso em face das tentativas de suicídio ${ }^{11,22,32-34}$. Esta eficácia, para alguns autores, se deve ao fato de que o enforcamento é o método mais realizado pelos homens, ao passo que as mulheres optam por meios mais passíveis de receberem atendimento e apoio para se recuperarem, como a ingestão de medicamentos ou intoxicação/envenenamento ${ }^{32,33,35}$. Contudo, no presente estudo, o enforcamento foi o meio mais utilizado também pelas mulheres, representando quase metade dos óbitos. Por outro lado, foram frequentes no sexo feminino óbitos ocorridos em hospitais ou em serviços de saúde, corroborando a literatura a respeito da escolha do método usado no ato suicida ${ }^{32,33,35}$.

Para alguns autores ${ }^{4,11}$, os meios utilizados no suicídio variam conforme a cultura e a disponibilidade. As principais causas de 
suicídio no estado foram enforcamento, autointoxicação por pesticidas e lesões por armas de fogo, representando mais de $80 \%$ das mortes, dados que se mostraram semelhantes aos encontrados no Brasil e no mundo $0^{4,7,11,32}$. Parte dessas mortes - lesões por armas de fogo, autointoxicação por pesticidas e medicamentos - poderia ser evitada e revela a necessidade de estabelecer ações preventivas para restringir o acesso a esses meios, como preconizado pela $\mathrm{OMS}^{4}$ e pelas Diretrizes Nacionais de Prevenção ao Suicídios ${ }^{28}$.

Assim como no Brasil ${ }^{1}$, quase a metade dos suicídios em Mato Grosso ocorreu na população de 20 a 39 anos. As taxas de mortalidade em adolescentes também são semelhantes às do Brasil, no entanto, as taxas em idosos se revelaram mais elevadas quando comparadas às do país ${ }^{1,5}$. Sobre os fatores associados à mortalidade por suicídio em idosos, alguns autores referem a depressão, o isolamento social, a perda do sentido da vida, as perdas de filhos e cônjuges, a presença de doenças graves e degenerativas, as privações econômicas e de afeto, a inatividade e as vivências de violências no passado e no momento presente da vida ${ }^{36-39}$. Berzins e Watanabe ${ }^{36}$ apontam que os idosos usam meios mais letais do que as pessoas mais jovens, resultando, quase sempre, na fatalidade do ato. Essas autoras $^{36}$ ainda destacam a relevância desses dados em face do crescimento acelerado do número de idosos no Brasil.

A maioria das pessoas que cometeram suicídio tinha baixa escolaridade (menos de 8 anos de estudo), assim como observado por outros autores ${ }^{7,40,41}$. O nível educacional, a situação de desemprego e a renda familiar definem o status econômico e social do indivíduo, o que proporciona distintos níveis de preocupações e estresse ${ }^{40}$.

O coeficiente de mortalidade por suicídio do estado pode esconder importantes variações intermunicipais. Estudos realizados nas duas últimas décadas mostram taxas mais elevadas em indígenas e em cidades de pequeno e de médio porte populacional $\left.\right|^{11,16,17}$, apontando a necessidade de desagregação dos resultados aqui aportados.

Importante limitação deste, assim como de outros estudos sobre suicídios no Brasil, deve-se à subestimação dos dados registrados pelo Sistema de Informação de Mortalidade do Ministério da Saúde ${ }^{11}$ ou à possibilidade dos suicídios serem registrados como outra causa de morte tal como referido na literatura ${ }^{11,18,19}$.

Além dos dados revelados neste estudo, é necessária a investigação dos fatores predisponentes, o que se recomenda para pesquisas futuras. Entre os diversos fatores (causas), os transtornos mentais se sobressaem, estando presente na maioria dos $\operatorname{casos}^{4,5,8,10,11,22,23}$, destacando-se a depressão, o transtorno bipolar, a esquizofrenia, o abuso de álcool e outras $\operatorname{drogas}^{22,23,25}$.

Neste sentido, o cuidado com os portadores de transtornos mentais, usuários de álcool e outras drogas e, principalmente, com os sujeitos que já tentaram suicídio anteriormente deve ser uma premissa para os serviços de saúde. Portanto, uma rede de atenção organizada e com profissionais qualificados deve ser fortalecida ${ }^{20-23}$. Em Mato Grosso, apesar do crescimento da rede de saúde mental nos últimos anos, a cobertura dos serviços é baixa, revelando-se muito inferior ao observado para o país ${ }^{30}$, denotando a necessidade de investimentos na área, visando à prevenção do suicídio no estado.

Sob este aspecto, Botega et al..$^{22}$ afirmam que, no Brasil, mesmo com a instituição do plano nacional de prevenção do suicídio ${ }^{27}$, pouco se fez em termos institucionais em relação à prevenção do ato suicida.

Falar sobre suicídio é também uma estratégia de prevenção. É essencial o incentivo à pesquisa sobre o suicídio e necessário que o tema seja pautado apropriadamente na mídia, nas escolas e nas instituições diversas, instigando a reflexão e o debate entre os gestores, os profissionais de saúde e a população em geral.

\section{CONCLUSÃO}

O estudo revela informações pouco exploradas sobre o suicídio no estado de Mato Grosso e, portanto, relevantes para ampliar o conhecimento sobre o problema e sua prevenção.

Apesar da estabilidade das taxas de mortalidade por suicídio ao longo dos anos, elas se apresentaram bem mais elevadas que o país, sugerindo que ações de prevenção são pouco efetivas. Há necessidade de outras pesquisas sobre suicídio no estado, especialmente aquelas que apontem fatores de risco. Contudo, os resultados aportados por este estudo indicaram alguns fatores envolvidos na gênese do suicídio, como a alta taxa de mortalidade em idosos e os principais meios utilizados no ato suicida.

Considerando a complexidade que envolve a ocorrência do suicídio, é necessário estabelecer estratégias intersetoriais de combate à sua redução. Espera-se, portanto, que este estudo contribua para a reflexão e para o planejamento das ações de prevenção do suicídio no estado.

\section{REFERÊNCIAS}

1. Ministério da Saúde [BR]. Saúde Brasil 2014: uma análise da situação de saúde e das causas externas. Brasília: Ministério da Saúde; 2015.

2. Ferreira A Jr. O comportamento suicida no Brasil e no mundo. Rev Bras Psicologia. 2015; 2(1):15-28.
3. World Health Organization. Suicide prevention and special programmes. Genebra: WHO; 2005

4. World Health Organization. Preventing suicide: a global imperative[internet]. Genebra: WHO; 2014 [acesso 2016 Jan 5]. Disponível em: http://apps.who.int/ 
iris/bitstream/10665/131056/8/9789241564878_eng.pdf?ua=1\&ua=1.

5. World Health Organization. The World Health Report 2001. Mental Health: New Understanding, New Hope [internet]. Genebra: WHO; 2001 [acesso 2016 Jan 3]. Disponível em: http://www.who.int/whr/2001/en/.

6. Durkheim E. O suicídio. Rio de Janeiro: Zahar; 1992.

7. Machado DB, Santos DN. Suicídio no Brasil, de 2000 a 2012. J Bras Psiquiatr. 2015 Jan-Mar; 64(1): 45-54. doi: http://dx.doi.org/10.1590/00472085000000056.

8. Organização para a Cooperação e Desenvolvimento Econômico. Society at a Glance 2014: The crisis and its Aftermath [internet]. Paris: OECD; 2014 [acesso 5 Jan 2016]. Disponível em: http://www.oecd.org/els/soc/OECD2014SocietyAtAGlance2014.pdf.

9. World Health Organization. The word health report. Shaping the future [internet]. Genebra: WHO; 2003 [acesso 2016 Jan 5]. Disponível em: http:// www.who.int/whr/2003/en/.

10. Bertolote JM, Fleischmann A. Suicide and psychiatric diagnosis: a worldwide perspective. World Psychiatry. 2020;1(3): 181-185

11. Botega NJ. Comportamento suicida: epidemiologia. Psicologia USP. 2014; 25(3): 231-236. doi: http://dx.doi.org/10.1590/0103-6564D20140004.

12. Botega NJ, Barros MBA, Oliveira HB, Dalgalarrondo P, Marín-León L. Suicida Behavior in the community: prevalence and factors associated to suicidal ideation. Rev Bras Psiquiatr. 2005; 27(1): 45-53. doi: http://dx.doi.org/10.1590/ S1516-44462005000100011.

13. Chachamovich E, Stefanello S, Botega NJ, Turecki G. Quais são os recentes achados clínicos sobre a associação entre depressão e suicídio? Rev Bras Psiquiatr. 2009; 31(1):18-25. doi: http://dx.doi.org/10.1590/S151644462009000500004

14. Krug EG, Dahlberg LL, Mercy JÁ, Zwi AB, Lozano R. Relatório mundial sobre violência e saúde[internet]. Genebra: WHO; 2002 [acesso 2016 Jan 6]. Disponível em: http://apps.who.int/iris/bitstream/10665/42495/1/9241545615_eng.pdf. doi: http://dx.doi.org/10.1590/S1516-44462009000500004

15. Waiselfisz JJ. Os jovens do Brasil: mapa da violência. Brasília: Ministério da Saúde; 2014.

16. Marín-León L, Oliveira HB, Botega NJ. Suicide in Brazil, 2004-2010: The importance of small counties. Rev Panam Salud Publica. 2012 Nov; 32(5): 351359. PubMed PMID: 23338692

17. Minayo MC, Pinto LW, Assis SG, Cavalcante FG, Mangas RMN. Trends in suicide mortality among Brazilian adults and elderly, 1980 - 2006. Rev Saude Publica. 2012; 46(2): 300-309. doi: http://dx.doi.org/10.1590/S003489102012000200012

18. Gotsens M, Olmo MMD, Sanz MR, Martos D, Espelt A, Pérez G, et al. Validation of the underlying cause of death in medicolegal deaths. Rev Espanhola Salud Publica. 2011; 85(2): 163-174. doi: 10.1590/S1135-57272011000200005.

19. Pritchard C, Hean S. Suicide and undetermined deaths among youths and young adults in Latin America. Comparison with the 10 major developed countries-A source of hidden suicides? Crisis. 2008; 29(3): 145-153. doi: 10.1027/0227-5910.29.3.145

20. Organização Mundial da Saúde. Prevenção do suicídio: um manual para profissionais da saúde em atenção primária [internet]. Genebra, OMS; 2000a [acesso 2016 Jan 4]. Disponível em: http://www.who.int/mental_health/ prevention/suicide/en/suicideprev_phc_port.pdf.

21. Organização Mundial da Saúde. Prevenção do suicídio: um manual para médicos clínicos gerais [internet]. Genebra: OMS; 2000 [acesso 2016 Jan 4]. Disponivel em: http://apps.who.int/iris/bitstream/10665/67165/7/WHO
MNH_MBD_00.1_por.pdf.

22. Botega NJ, Bertolote JM, Hetem LA, Bessa MA. Prevenção do Suicídio. Debates: Psiquiatr Hoje. 2010; 2(1): 10-20.

23. Ministério da Saúde [BR]. Prevenção do Suicídio: Manual dirigido a profissionais das equipes de saúde mental. Brasília: Ministério da Saúde; 2006a.

24. Botega NJ, Garcia LSL. Brazil: the need for violence (including suicide) prevention. World Psychiatry. 2004; 3(3): 157-158. PubMed PMID: 16633484

25. Kohlrausch E, Lima MADS, Abreu KP, Soaes JSF. Atendimento ao comportamento suicida: concepções de enfermeiras de Unidade de Saúde. Cienc Cuid Saude. 2008; 7(4): 468-475.

26. Machin R. Nem doente, nem vítima: o atendimento às "lesões autoprovocadas" nas emergências. Cien saúde coletiva. 2009; 14(5): 17411750. doi: http://dx.doi.org/10.1590/S1413-81232009000500015.

27. Kondo EH, Vilella JC, Borba LO, Paes MR, Maftum MA. Abordagem da equipe de enfermagem ao usuário na emergência em saúde mental em um pronto atendimento. Rev esc enferm. USP. 2011; 45(2): 501-507. doi: http://dx.doi. org/10.1590/S0080-62342011000200028.

28. Ministério da Saúde [BR]. Portaria no1.876 de 14 de Agosto de 2006. Institui Diretrizes Nacionais para Prevenção do Suicídio, a ser implantadas em todas as unidades federadas, respeitadas as competências das três esferas de gestão [internet].Diário Oficial [da] República Federativa do Brasil. 2006 Ago 18 [acesso 2016 Jan 5]. Disponível em: http://bvsms.saude.gov.br/bvs/saudelegis/ gm/2006/prt1876_14_08_2006.html.

29. Organização Mundial da Saúde. Centro Colaborador da OMS para a Classificação de Doenças em Português. Classificação Estatística Internacional de Doenças e Problemas Relacionados à Saúde: 10ạ Revisão (CID-10). 9 ed. São Paulo: EdUSP; 2003.

30. Ministério da Saúde [BR]. Departamento de Informática do Sistema Único de Saúde - DATASUS [internet]. Brasília: 2016 [acesso 2016 Jan 3]. Disponível em: http://www2.datasus.gov.br/DATASUS/index.php?area=02

31. Mato Grosso. Secretaria de Estado da Saúde - SES. [internet]. Cuiabá: 2017 [acesso 2017 Jan 4]. Disponível em: http://appweb3.saude.mt.gov.br/dw/ pesquisa/detalhe $O$ link informado nesta referência é o principal do repositório da Secretaria de Saúde de MT, mas qual o link específico do texto que foi utilizado?

32. Batista MN, Carneiro AM, Gomes JO, Cardoso HF. Análise Epidemiológica do Suicídio em duas Regiões do Estado de São Paulo entre 2004 e 2008. Psicol. pesq. 2012; 6(2): 02-12

33. Lovisi GH, Santo SA, Legay L, Abelha L, Valencia E. Análise epidemiológica do suicídio no Brasil entre 1980 e 2006. Rev Bras Psiquiatr. 2009; 31(Supl. II): 86-93. doi: http://dx.doi.org/10.1590/S1516-44462009000600007.

34. Medina Pérez O, Piernagorda DC, Rengifo AJ. Prevalencia del suicidio en adultos jóvenes en el departamento del Quindío - Colombia, entre 1989 y 2008. Pensamiento Psicológico. 2010; 8(15): 9-16.

35. González A. Betancur AR, Aristizäbal A, Valencia JG, Palacio C, Jaraillo CL. Suicidio y género en Antioquia (Colombia): Estudio de autopsia psicológica. Rev Colomb Psiquiatr. 2010; 39(2): 251-267.

36. Berzins MV, Watanabe HAW. Falar de suicido é também falar da vida e da qualidade de vida. Cien saúde coletiva. 2012; 17(8): 1955-1962.

37. Conwell, Y. Challenges to preventing suicide in later life. Cien saude Colet. 2015; 20(6): 652-653. doi: http://dx.doi.org/10.1590/141381232015206.1996201

38. De Leo D, Padoani W, Scocco P, Lie D, Bille-Brahe U, Arensman E, et al. Attempted and completed suicide in older subjects: results from the WHO/ 
EURO multicentre study of suicidal behaviour. Int J Geriatr Psychiatry. 2001; 16(3): 300-310. PubMed PMID: 11288165

39. Minayo MC, Cavalcante FG. Suicide in elderly people: a literature review. Rev Saude Publica. 2010; 44(4): 750-757. doi: http://dx.doi.org/10.1590/S0034 89102010000400020

40. Desaulniers J, Daigle MS. Inter-regional variations in men's attitudes, suicide rates and so-ciodemographics in Quebec (Canada). Soc Psychiatry Psychiatr Epidemiol. 2008 Jun; 43(6): 445-453. doi: 10.1007/s00127-008-0340-2.

41. Stevovic LI, Jašović-Gašić $M$, Vukovic $O$, Peković $M$, Terzić N. Gender differences in relation to suicides committed in the capital of Montenegro (Podgorica) in the period 2000-2006. Psychiatr Danub. 2011 Mar; 23(1): 45-52. PubMed:21448096.

\section{Como citar este artigo/How to cite this article:}

Oliveira LR, Benedetti AOC. Suicídio em Mato Grosso - Brasil: 1996 a 2015. J Health Biol Sci. 2018 Out-Dez; 6(4):91-398. 